\title{
Osteoblastic and Vascular Endothelial Niches, Their Control on Normal Hematopoietic Stem Cells, and Their Consequences on the Development of Leukemia
}

\author{
Bella S. Guerrouahen,, ${ }^{1,2}$ Ibrahim Al-Hijji, ${ }^{3,4}$ and Arash Rafii Tabrizi ${ }^{1,2,3}$ \\ ${ }^{1}$ Department of Genetic Medicine, Weill Cornell Medical College, New York, NY 10022, USA \\ ${ }^{2}$ Stem Cell and Microenvironment Laboratory, Weill Cornell Medical College in Qatar, Education City, P.O. Box 24144, Doha, Qatar \\ ${ }^{3}$ Hamad Medical Corporation, Doha, Qatar \\ ${ }^{4}$ Medical Oncology Department, Al Amal Hospital, Hamad Medical Corporation, Doha, Qatar
}

Correspondence should be addressed to Arash Rafii Tabrizi, jat2021@qatar-med.cornell.edu

Received 1 June 2011; Accepted 19 October 2011

Academic Editor: Linheng Li

Copyright $\odot 2011$ Bella S. Guerrouahen et al. This is an open access article distributed under the Creative Commons Attribution License, which permits unrestricted use, distribution, and reproduction in any medium, provided the original work is properly cited.

Stem cell self-renewal is regulated by intrinsic mechanisms and extrinsic signals mediated via specialized microenvironments called "niches." The best-characterized stem cell is the hematopoietic stem cell (HSC). Self-renewal and differentiation ability of HSC are regulated by two major elements: endosteal and vascular regulatory elements. The osteoblastic niche localized at the inner surface of the bone cavity might serve as a reservoir for long-term HSC storage in a quiescent state. Whereas the vascular niche, which consists of sinusoidal endothelial cell lining blood vessel, provides an environment for short-term HSC proliferation and differentiation. Both niches act together to maintain hematopoietic homeostasis. In this paper, we provide some principles applying to the hematopoietic niches, which will be useful in the study and understanding of other stem cell niches. We will discuss altered microenvironment signaling leading to myeloid lineage disease. And finally, we will review some data on the development of acute myeloid leukemia from a subpopulation called leukemia-initiating cells (LIC), and we will discuss on the emerging evidences supporting the influence of the microenvironment on chemotherapy resistance.

\section{Introduction}

Stem cells are self-renewing, and multipotent or pluripotent cells present in different compartments of the body, responsible for the growth, maintenance, and repair of the different tissues. The stem cells reside in a special microenvironment; the so-called "niche," composed of cellular structures and extracellular matrix in which those cells are tightly regulated regarding their balance between maintenance of stemness, expansion, and differentiation [1].

One of the best characterized stem cell is the hematopoietic stem cells (HSC) and in 1978, Schofield proposed the "niche" hypothesis to the HSC bone marrow (BM) microenvironment [2]. HSCs function to generate a lifelong supply of all blood cell types. The ability to reconstitute all the bloodcell lineages in lethally irradiated mice is the most common assay to assess the stemness in HSC [3]. And furthermore, the maintenance of this property upon serial transplantation shows the capability of these cells to undergo self-renewal and to give rise to a differentiated progeny at the same time. In the past few years, the microenvironment, which regulates HSCs, has been characterized. Within adult BM, self-renewal and differentiation are regulated by two major cellular components: osteoblasts and vascular endothelial cells [4]. In this review, we provide some principles applying to the hematopoietic niches, which will be useful in the study and understanding of other stem cell niches. Furthermore, the involvement of the niches in the development of leukemia and its resistance to treatment will be described.

\section{The Osteoblastic Niche}

The concurrence of hematopoiesis and osteogenesis in BM suggests a close relationship between bone-forming cells and hematopoietic cells [5]. 
2.1. In Vivo Evidence of the Osteoblastic Niche. In the BM, quiescent or slow-cycling HSC have been identified close to the endosteal surface in the trabecular bone; constituting a reservoir of HSC that can be mobilized and restore the hematopoiesis in response of tissue injury. Celso and collaborators demonstrated that after transplantation, an HSC pool was observed in the area closest to the endosteum. They also showed that as differentiation progressed, they homed into distinct locations [6]. Xie and collaborators showed that transplanted HSCs were homing to the endosteum in the $\mathrm{BM}$, which maintains and promotes HSC [7]. We have in vivo evidence of an osteoblastic niche, which might serve as a reservoir for HSC storage.

2.2. Secreted Factors. The bone-forming osteoblasts are crucial players for the homeostasis of the hematopoietic tissue with its high turnover. Indeed, osteoblasts express several cell-signaling molecules such as BMP4, Jagged-1, and angiopoietin-1 (Ang1), which are important for HSC selfrenewal, survival, and maintenance $[8,9]$ and have been proposed to control HSC fate. Ang1 in osteoblasts interacts with Tie-2, a type of receptor tyrosine kinase that is expressed in HSC, and the Tie-2/Ang1 interaction activates $\beta 1$-integrin and $\mathrm{N}$-cadherin. This enhanced adhesion contributes to the maintenance of the stem cell quiescence. Osteopontin, a matrix glycoprotein expressed by the osteoblasts supports the adhesion of HSC to the osteoblastic niche and negatively regulates HSC proliferation, contributing to the maintenance of a quiescent state [10]. Furthermore, osteoblasts in the bone marrow produce a significant amount of membrane-bound stem cell factor (SCF), which has the capacity to enhance the adhesion of HSC to stromal cells [11], suggesting that osteoblasts can influence the adhesive characteristics of the endosteal niche by modulating the functional state of specific integrins. SCF binds and activate c-KIT, which is highly expressed by label retaining HSC. It has been previously shown that mutations which affect the membrane bound SCF have an influence on migration and differentiation of different cells including hematopoietic cells [12]. In addition a high concentration of $\mathrm{Ca}^{2+}$ in the $\mathrm{BM}$ endosteal region mediates HSC homing and lodging in the osteoblastic niche by attracting HSCs through the calcium sensor receptor that is highly expressed in HSCs [13].

2.3. Membrane-Bound Factors. In 2003, the work leaded by two research groups supported the idea that osteoblasts control the number HSC by regulating the size of the niche. Calvi and his collaborators were able to show a direct contact between osteoblasts and HSCs in some in vivo studies using mutants, with increased or decreased number of osteoblasts [14]. This study showed that an increase in the number of osteoblasts by administration of parathyroid hormone (PTH) produce high levels of notch ligand Jagged-1 and increase the number of HSC. Zhang and his collaborators showed also that it was possible to increase or decrease the HSC number by modulating the osteoblast number. They also showed that HSC and osteoblasts bind each other via adhesion molecules such as
$\mathrm{N}$-cadherin. Although not all osteoblasts function as the HSC niche, and a subset expressing a high level of N-Cadherin function as a key component. These cells expressing a high level of $\mathrm{N}$-Cadherin are located in the bone surface and named: spindle-shaped N-cadherin+CD45- osteoblastic cells (SNO) [15]. Wilson and collaborators found that the majority of HSCs/progenitors lodge in the endosteum and attach to $\mathrm{N}$-Cadherin + osteoblastic cells 15 hours after transplantation, and HSCs harboring a c-Myc mutation increases $\mathrm{N}$-Cadherin expression, enhancing adhesion to osteoblasts [16]. However, the fact that hematopoiesis appears normal in N-cadherin-deficient mice [17] and the localization of a significant amount of HSC in proximity of the sinusoidal walls in the BM support the hypothesis of another specialized microenvironment affecting HSC regulation: the vascular niche $[18,19]$.

\section{The Vascular Niche}

Some evidences about the existence of a vascular niche were already present, since in case of injury HSC can detach from the endosteum and migrate to the vascular region of the BM from where they restore hematopoiesis [20]. The vascular niche promotes proliferation, differentiation of actively cycling, and short-term HSCs [21]. The most purified HSCs, fractioned as CD150+ CD48- CD41- Lincells, were found in majority to be associated with the sinusoidal endothelium lining blood vessels, suggesting that endothelial cells create a cellular niche for HSCs [18].

\subsection{Hemangioblasts Differentiate toward HSC and Endothe-} lial Cells. A close developmental relationship between the hematopoietic and the endothelial lineages has been shown in the study led by Keller's group [22]. HSCs seem to originate from a perivascular progenitor during embryonic development. Moreover, the presence of HSC in the yolk sac, aorta-gonad-mesonephros (AGM) region and vitelline arteries suggests that HSCs reside and undergo hematopoiesis in association with blood vessels. HSCs are found in close contact with endothelial cells at any time during development. The endothelial cells harvested from these regions have been shown to support the expansion of adult HSC LSK (Lineage ${ }^{-} \mathrm{Sca}-1^{+} \mathrm{c}-\mathrm{Kit}^{+}$) cells in vitro $[23,24]$. In contrast, endothelial cells isolated from adult organs do not display an HSC supportive activity in vitro [25]. These observations support a strong embryologically interdependence between HSCs and endothelial cells of BM sinusoids and indicate that these cells hold distinct functional characteristics from endothelial cells present in other tissues.

3.2. In Vivo Evidence of the Vascular Niche. On the one hand, conditional deletion of vascular endothelial growth factor receptor 2 (VEGF2) in adult mice, inhibits regeneration of sinusoidal endothelial cells, and prevents hematopoietic reconstitution in irradiated animals [26]. And, on the other hand, thrombopoietin knock-out mice used by Rafii's group showed remarkably that CXCL12 (SDF-1, stromal cellderived factor-1) and FGF-4 (fibroblast growth factor-4) promotes megakaryocytopoiesis, restoring platelet production. 
Among other things, SDF-1 and FGF-4 induced upregulation of adhesion molecules, including VLA4/VCAM1, facilitating localization to the vascular niche [27].

3.3. Secreted Factors. The vascular niche has been shown to produce factors important for mobilization, homing, and engraftment of HSC, such as SDF-1 important for mobilization, homing, and engraftment for HSCs [27]. The role of primary human endothelial cells (PECs) in the regulation of hematopoiesis, has been highlighted in 2006 by a study led by Yeoh et al. [28]. This research group showed that the cell-autonomous capacity to support homeostasis was hidden in the presence of serum and angiogenic factors (VEGF-A and FGF2), essential to maintain PECs for coculture with hematopoietic stem progenitors (HSPs). To overcome this issue, Rafii's group generated a model to study the vascular niche, where endothelial cells could propagate, while maintaining their long-term angiogenic profile without oncogenic transformation. Indeed, they transduced human primary endothelial cells (PECs) with the adenovirus gene, early region 4 encoded open reading frame1 (E4ORF1) [29], which leads to constitutive activation of Akt that allows for long-term culture. E4ORF1-transduced PECs expand HSCs in vitro through secretion of stem cell active angiocrine factors that sustain self-renewal of KLS cells [30]. Selective activation of Akt in the endothelial cells of adult mice increases the number of HSCs and accelerates hematopoietic recovery after myeloablation along with upregulation of a specific set of hematopoietic stem and progenitor cells (HSPCs) angiocrine factors, like FGF2, BMP4, Ang1, IGFBP2, and DHH [31].

3.4. Membrane-Bound Factors. During the bone marrow transplantation, the ability of HSCs to "home" and engraft in the recipient's bone marrow requires a cascade of events which includes specific molecular recognition. Adhesion molecules on the HSCs involved in the process of rolling are VLA4 (CD49d), LFA-1 (CD11a), and hyaluronan bindingcellular adhesion molecule (HCAM/CD44) whereas the complementary binding partners on the BM endothelial cells are VCAM-1, ICAM-1 (CD54), and E- and P-selectin (CD62E and CD62P) [32]. The notch signaling pathway involves the activity of notch transmembrane receptors 1,3 , and 4, which interact with membrane-bound ligands, Delta1, 2 , and 4, and Serrate/Jagged-1 and 2. Ligand binding induces proteolytic cleavage of notch receptor by a gamma-secretase complex causing the subsequent translocation of the notch intracellular domain (NICD) to the nucleus, where it will activate the transcription of downstream target genes such as Hes1 (hairy enhancer of split homolog-1) and Hey1 and Hey2 (Hes-related protein) [33]. Notch-1 is expressed on LT-HSCs and maintains HSCs in an undifferentiated state $[14,34,35]$. Overexpression of the intracellular domain of notch enhances HSC self-renewal [36] whereas loss of notch signaling impairs the maintenance of HSCs both in vitro and in vivo [14, 37]. Butler and his collaborators blocked angiogenic pathways, which led to inhibition of notch ligand expression and a decrease of LT-HSCs expansion [30].
Altogether, these observations suggest that endothelial cells enhance proliferation of HSCs and play an important role in maintaining the pool.

\section{Common Components and Differences}

CXCL12/CXCR4 signaling plays important roles in HSC trafficking and HSC mobilization. Sugiyama and his collaborators [38] demonstrated that HSCs were specifically located adjacent to cells expressing a high level of CXCL12, which surrounded the sinusoidal endothelial cells. They named these cells CXCL12-abundant reticular cells (CAR). They also demonstrated that a depletion of CXCR4 led to a reduction of HSC pool. Selective short-term ablation of CAR cells within bone marrow has suggested that they play a crucial role in proliferation, maintenance in an undifferentiated state. HSCs have been shown to be associated with CAR cells in the sinusoidal region and are located between CAR and osteoblastic cells in the endosteal region, suggesting that those cells might be an important component of both the osteoblastic and the vascular niches in adult BM.

The major difference between both microenvironments is the oxygen level. Higher in the vascular niche than in the osteoblastic niche under hypoxia, HSCs would move to the vascular niche and resume then cell cycle in order to restore hematopoiesis [39]. HSCs can then come back to the osteoblastic niche where they would be maintained in the G0 state once again [40]. The role of the hypoxia has recently been demonstrated. Using a transgenic $\mathrm{VEGF}^{\delta / \delta}$ mice model where the hypoxia response element in the VEGF promoter is enable to bind HIF; the authors demonstrated impairment of HSC function and confirmed that one important role of the hypoxic niche is to provide HSCs intrinsically with the survival factor VEGFA [41].

The osteoblastic niche localized at the inner surface of the bone cavity and with abundant osteoblasts, might serve as a reservoir for long-term HSC storage in a quiescent state. Whereas the vascular niche, which consists of sinusoidal endothelial cell lining blood vessel, provides an environment for short-term HSC proliferation and differentiation. Both niches act together to maintain hematopoietic homeostasis or restore it after damage.

\section{Altered Microenvironment Leads to Myeloid Lineage Diseases}

Several studies have provided insights into the role of altered microenvironment signaling leading to myelofibrosis, myeloma, and myelodysplastic syndromes. It has been shown that alterations of the microenvironment, such as decreased expression of the retinoblastoma gene in cells of the BM microenvironment, can induce a myeloproliferative disorder [42]. Latillade reviews key data suggesting that an imbalance between endosteal and vascular niches may be important in idiopathic disorder characterized by bone marrow fibrosis (primary myelofibrosis), leading to the development of clonal stem cell proliferation [43]. 
Wnt signaling pathway plays a critical role in the pathogenesis of myeloma. A dramatic effect of directly targeting this pathway in osteoblasts has been clearly demonstrated, showing that it could prevent in vivo, the development of myeloma disease within bone. Increasing Wnt signaling in the bone marrow microenvironment inhibits the development of myeloma and reduces tumor burden in bone in vivo [44]. Microenvironment perturbations play also a role in myelodysplastic syndromes (MDS). Raaijmakers and his collaborators have developed transgenic mice with a dicer knock out only in osteoblast progenitor to investigate if altered niche caused myelodysplasia [45]. Although dicer, which encodes a critical enzyme in the formation of microRNAs, was not deleted from the hematopoietic cells, these mice developed a form of myelodysplastic disease. To identify the mechanism by which dicer deletion in osteoblast progenitors may cause MDS, the investigators performed gene expression analysis. Differentially expressed genes and pathways included cytokines and stress response pathways, including significant downregulation of the ShwachmanBodian-Diamond (sbds) gene, which is linked to the human Shwachman-Diamond syndrome. Although the mechanism linking dicer deletion to sbds expression is not clear, the investigators showed that knockdown of just the Sbds gene in osteoblast progenitors reproduces much of the MDS phenotype.

\section{Consequences of the Niches in the Development of Leukemia and Resistance to Treatment}

AML is the most common leukemia according to the SEER program of the National Cancer institute. AML is a cancer of the myeloid line of blood cells. AML is a stem cell disease with a hierarchy comparable to normal hematopoiesis. In parallel with leukemogenesis events, the niche provides an environment with signals that favor cell proliferation and growth. Although, recent line of evidences suggest that the tumor cells are heterogeneous and indicate that only a fraction of the tumor cells "the leukemia-initiating cells" (LIC) are mostly in a quiescent state and are responsible for the maintenance of the neoplasm [46].

6.1. In Vivo Evidence of Niches for Leukemia Cells. It has been demonstrated that the normal hematopoiesis disappear in leukemic patients, disturbing the BM niches [47]. Both niches play a critical role in the survival, proliferation, and differentiation of LSC. The group led by Ninomiya showed the homing proliferation and survival sites of leukemia cells in immunodeficiencies mice. Indeed, when transplanted, leukemia cells initially localized on the surface of the osteoblasts in the inner vascular and diaphyseal region. Under administration of a high dose of cytarabine leukemia cells clustered and adhered to the blood vessels suggesting that leukemia cells receive antiapoptotic signals from vascular niche as well [48]. The molecular mechanisms for maintaining quiescence of normal HSCs may also facilitate LSC survival.
6.2. Secreted Factors. Similar to regeneration of hematopoietic cells, leukemic cells may also take advantage of prosurvival signals conveyed by the osteoblastic and vascular niches to support their proliferation and invasive potential. Osteoblasts provide a source of osteopontin and SDF-1, which may induce migration of CXCR4-expressing LSCs towards the osteoblastic niche. The interaction between SDF1 and its receptor on leukemic progenitor cells contributes to their homing to the BM microenvironment. Endothelial cells are protective of leukemia and increased levels of angiogenesis in the BM of AML patients have been reported [49]. Moreover VEGF acts as an autocrine and paracrine growth factor in some AMLs that express the VEGFR2 [50, 51]. VEGF is a hypoxia-inducible growth factor that stimulates the formation of new blood vessels. As an adaptive response to hypoxia, new vessels are generated by angiogenesis and the proliferation of endothelial cells is stimulated. By secretion of cytokines, including IL-6, IL-3, G-CSF, GM-CSF, and nitric oxide (NO) $[52,53]$, endothelial cells were shown to promote leukemic proliferation, suggesting that activated angiogenic endothelial cells could provoke leukemic cell growth through paracrine signaling.

6.3. Membrane Bound Factors. The attachment of AML cells to the $\mathrm{BM}$ microenvironment through interaction between very late antigen- 4 on leukemic cells and fibronectin on stromal cells has been shown to be crucial for the persistence of minimal residual disease in AML [54]. CXCR4 levels are significantly elevated in leukemic cells from patients with AML [55] and CXCR4 expression is associated with poor outcome $[56,57]$. It has been shown that administration of anti-CXCR4 antibody to a NOD/SCID mice engraft with primary AML cells resulted in a dramatic decrease in the level of human AML cells in different sites/BM, blood, and spleen. Interestingly, the levels of normal human progenitor cells were not affected [58]. Dick and colleagues showed that anti-CD44 antibody-treated NOD/SCID mice transplanted with AML exhibited a significantly lower rate of disease onset [59]. This indicates that CD44 is essential for the homing and engraftment of the LSC to the niche. To summarize the role of the microenvironment in leukemia and its normal physiologic role in supporting HSC it has been summarized in Figure 1.

6.4. Microenvironment and Resistance to Chemotherapy. From a clinical perspective, it has been demonstrated that LSCs are substantially more resistant to standard forms of chemotherapy than bulk leukemia populations. In order to optimize the treatment, it is mandatory to characterize the interaction of leukemic cells with their microenvironment in the bone marrow. The tumor microenvironment is increasingly being recognized as a critical factor in mediating drug resistance. Resistance may occur through the soluble release of growth factors and by contact-dependent mechanisms via cell-cell and cell-extracellular matrix interactions. The term, cell adhesion-mediated drug resistance (CAM-DR) has been used to describe the phenotype in which interaction of malignant cells with stroma confers resistance to chemotherapy. CAM-DR has been described in vitro for both primary 


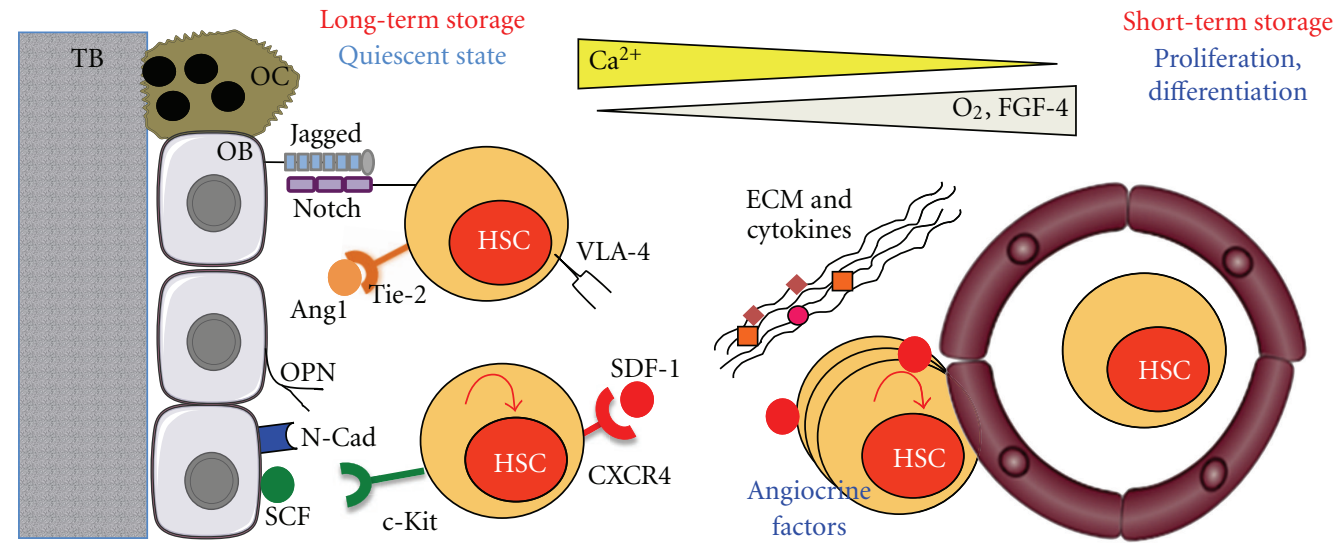

(a)

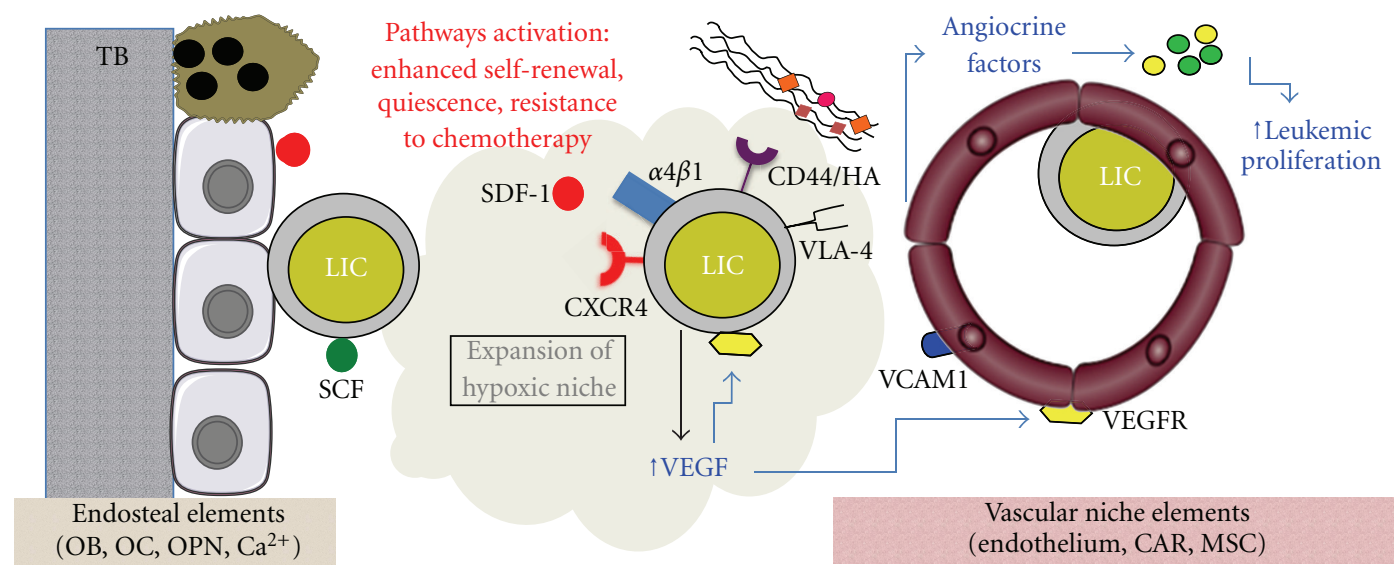

(b)

FIGURE 1: Schematic representation of the (a) HSC, normal stem cell and (b) LIC, leukemia-initiating cell interactions with the microenvironment. Niches provide support for self-renewal, quiescence, homing, enfrafment, and proliferation. As shown, HSCs and LSC reside in the osteoblastic or vascular niche. HSCs and LSC interact with multiple cell types (OB, OC, EC, CAR, MSC) within the $\mathrm{BM}$ microenvironment. Although schematically represented separately, the endosteal, and vascular niches are physically and functionally mutually involved. Candidate niche mechanisms, which regulate HSC function are also shown, including Jagged/notch, SDF1/CXCR4, $\mathrm{SCF} / \mathrm{c}-$ Kit signaling. LIC quiescence, survival, and expansion are influenced by receptor kinases, adhesive receptors and signaling via matrix mediated/bound chemokines and cytokines (IL6, G-CSF, GM-CSF, KIT L, NOTCH L). LIC may secrete substance like SCF, infiltrate the niches, and take advantage of the normal hemostatic process, which enhances self-renewal and proliferation. Growth factor and other adhesion receptors signals can be targeted to overcome chemoresistance. Oxygen level, higher in the vascular niche than the osteoblastic niche under hypoxia. LIC proliferation results in expansion of hypoxic niche. HSC: hematopoietic Stem Cell, OPN: osteopontin, LIC: leukemiainitiating Cell, N-Cad: N-Cadherin, OC: osteoclast, VLA-4: very late antigen 4, OB: osteoblast, VEGFR: vascular endothelial growth factor receptor, TB: trabecular bone, SDF1: stromal growth factor 1, SCF: stem cell factor, Ang1: angiopoietin 1, HA: hyaluronic acid, ECM: extracellular matrix.

tumors and cell lines derived from several hematologic malignancies [60]. There is an increase in neovessel density in the marrow of patients with leukemia; suggesting that crosstalk between leukemic and the activated vascular niche contribute to disease progression. Dr. Rafii and others have shown that inhibition of angiogenesis effectively blocks the progression of subsets of leukemias. Clinical trials have been initiated to evaluate the use of antiangiogenic agents in combination with standard chemotherapy to treat leukemias.

The hematopoietic system continues to evolve as a model; however, concerning the microenvironment, numerous critical issues remain to be addressed. A critical role for notch in hematopoiesis was initially suggested after detection of the human NOTCH-1 gene in CD34+ or CD34+ lin- human hematopoietic precursors [61]. Then several reports [36, 62, 63 ] have shown that NOTCH-1 plays a key regulatory role in hematopoiesis and suggest that notch ligands will be useful reagents for improving ex vivo culture of stem/progenitor cells. In 2010, a research group leaded by Bernstein reports the development of an optimized, clinically feasible methodology for generating cord blood stem/progenitor cells for clinical evaluation. Ex vivo culture of CD34+ cord blood stem/progenitor cells in the presence of notch ligand resulted in a greater than 100-fold increase in CD34+ cells that 
repopulate immunodeficient mice. This work was the first demonstration of rapid hematopoietic engraftment derived from ex vivo expanded hematopoietic progenitors. A recent study has demonstrated for the first time that the activation of notch pathway may indicate a poor prognosis in $\mathrm{AML}$ [64]. Especially, NOTCH-1, Jagged-1 and Delta1 expression may be relevant prognostic markers in intermediate risk AML. Nowadays, the most clinically arguably advanced approach for influencing leukemia microenvironment is targeting the angiogenesis. For example, therapies combining a monoclonal antibody neutralizing VEGF, bevacizumab with other chemotherapies as (cytarabine and mitoxantrone) improve the overall response rate of relapse and refractory AML [65]. Recently, new antiangiogenic agents are in use; among them, tyrosine kinase inhibitors (such as sorafenib for targeting VEGFR) and anticytokine drugs (such as thalidomide). On the one hand, those therapies can affect vascular endothelial niche but on the other hand, they may induce chemoresistance through the increase of hypoxia. In conclusion, targeting leukemic cells and cells in their surrounding microenvironment might be useful to observe a reduction of the leukemic burden. Going forward, it is challenging to revisit many of the concepts derived from work in the blood field in other organ systems. This will probably increase our knowledge on the network controlling self-renewal and differentiation, the hallmarks of stem cells.

\section{Acknowledgment}

This paper was funded by NPRPs: 09-1174-3-291; NPR 08663-3-140, NPRP-09-21087-3-274.

\section{References}

[1] K. W. Orford and D. T. Scadden, "Deconstructing stem cell self-renewal: genetic insights into cell-cycle regulation," Nature Reviews Genetics, vol. 9, no. 2, pp. 115-128, 2008.

[2] R. Schofield, "The relationship between the spleen colonyforming cell and the haemopoietic stem cell. A hypothesis," Blood Cells, vol. 4, no. 1-2, pp. 7-25, 1978.

[3] L. E. Purton and D. T. Scadden, "Limiting factors in murine hematopoietic stem cell assays," Cell Stem Cell, vol. 1, no. 3, pp. 263-270, 2007.

[4] J. M. Perry and L. Li, "Disrupting the stem cell Niche: good seeds in bad soil," Cell, vol. 129, no. 6, pp. 1045-1047, 2007.

[5] H. M. Patt and M. A. Maloney, "Bone marrow regeneration after local injury: a review," Experimental Hematology, vol. 3, no. 2, pp. 135-148, 1975.

[6] C. L. Celso, H. E. Fleming, J. W. Wu et al., "Live-animal tracking of individual haematopoietic stem/progenitor cells in their niche," Nature, vol. 457, no. 7225, pp. 92-96, 2009.

[7] Y. Xie, T. Yin, W. Wiegraebe et al., "Detection of functional haematopoietic stem cell niche using real-time imaging," Nature, vol. 457, no. 7225, pp. 97-101, 2009.

[8] F. Arai, A. Hirao, M. Ohmura et al., "Tie2/angiopoietin-1 signaling regulates hematopoietic stem cell quiescence in the bone marrow niche," Cell, vol. 118, no. 2, pp. 149-161, 2004.

[9] R. S. Taichman, "Blood and bone: two tissues whose fates are intertwined to create the hematopoietic stem-cell niche," Blood, vol. 105, no. 7, pp. 2631-2639, 2005.
[10] S. K. Nilsson, H. M. Johnston, G. A. Whitty et al., "Osteopontin, a key component of the hematopoietic stem cell niche and regulator of primitive hematopoietic progenitor cells," Blood, vol. 106, no. 4, pp. 1232-1239, 2005.

[11] T. Kinashi, T. A. Springer, A. W. Nienhuis, and P. J. Quesenberry, "Adhesion molecules in hematopoietic cells," Blood Cells, vol. 20, no. 1, pp. 25-44, 1994.

[12] S. D. Lyman and S. E. Jacobsen, "c-kit ligand and flt3 ligand: stem/progenitor cell factors with overlapping yet distinct activities," Blood, vol. 91, no. 4, pp. 1101-1134, 1998.

[13] G. B. Adams, K. T. Chabner, I. R. Alley et al., "Stem cell engraftment at the endosteal niche is specified by the calciumsensing receptor," Nature, vol. 439, no. 7076, pp. 599-603, 2006.

[14] L. M. Calvi, G. B. Adams, K. W. Weibrecht et al., "Osteoblastic cells regulate the haematopoietic stem cell niche," Nature, vol. 425, no. 6960, pp. 841-846, 2003.

[15] J. Zhang, C. Niu, L. Ye et al., "Identification of the haematopoietic stem cell niche and control of the niche size," Nature, vol. 425, no. 6960, pp. 836-841, 2003.

[16] C. E. Wilson, J. D. de Bruijn, C. A. van Blitterswijk, A. J. Verbout, and W. J. A. Dhert, "Design and fabrication of standardized hydroxyapatite scaffolds with a defined macroarchitecture by rapid prototyping for bone-tissue-engineering research," Journal of Biomedical Materials Research A, vol. 68, no. 1, pp. 123-132, 2004.

[17] M. J. Kiel, M. Acar, G. L. Radice, and S. J. Morrison, "Hematopoietic stem cells do not depend on n-cadherin to regulate their maintenance," Cell Stem Cell, vol. 4, no. 2, pp. 170-179, 2009.

[18] M. J. Kiel, O. H. Yilmaz, T. Iwashita, O. H. Yilmaz, C. Terhorst, and S. J. Morrison, "SLAM family receptors distinguish hematopoietic stem and progenitor cells and reveal endothelial niches for stem cells," Cell, vol. 121, no. 7, pp. 1109-1121, 2005.

[19] M. J. Kiel, G. L. Radice, and S. J. Morrison, "Lack of evidence that hematopoietic stem cells depend on n-cadherin-mediated adhesion to osteoblasts for their maintenance," Cell Stem Cell, vol. 1, no. 2, pp. 204-217, 2007.

[20] F. Velardi, P. R. Amante, M. Caniglia et al., "Osteogenesis induced by autologous bone marrow cells transplant in the pediatric skull," Child's Nervous System, vol. 22, no. 9, pp. 11581166, 2006.

[21] H. G. Kopp, S. T. Avecilla, A. T. Hooper, and S. Rafii, "The bone marrow vascular niche: home of HSC differentiation and mobilization," Physiology, vol. 20, no. 5, pp. 349-356, 2005.

[22] T. L. Huber, V. Kouskoff, H. J. Fehling, J. Palis, and G. Keller, "Haemangioblast commitment is initiated in the primitive streak of the mouse embryo," Nature, vol. 432, no. 7017, pp. 625-630, 2004.

[23] W. Li, S. A. Johnson, W. C. Shelley et al., "Primary endothelial cells isolated from the yolk sac and para-aortic splanchnopleura support the expansion of adult marrow stem cells in vitro," Blood, vol. 102, no. 13, pp. 4345-4353, 2003.

[24] O. Ohneda, C. Fennie, Z. Zheng et al., "Hematopoietic stem cell maintenance and differentiation are supported by embryonic aorta-gonad-mesonephros region-derived endothelium," Blood, vol. 92, no. 3, pp. 908-919, 1998.

[25] W. Li, S. A. Johnson, W. C. Shelley, and M. C. Yoder, "Hematopoietic stem cell repopulating ability can be maintained in vitro by some primary endothelial cells," Experimental Hematology, vol. 32, no. 12, pp. 1226-1237, 2004. 
[26] A. T. Hooper, J. M. Butler, D. J. Nolan et al., "Engraftment and reconstitution of hematopoiesis is dependent on VEGFR2mediated regeneration of sinusoidal endothelial cells," Cell Stem Cell, vol. 4, no. 3, pp. 263-274, 2009.

[27] S. T. Avecilla, K. Hattori, B. Heissig et al., "Chemokinemediated interaction of hematopoietic progenitors with the bone marrow vascular niche is required for thrombopoiesis," Nature Medicine, vol. 10, no. 1, pp. 64-71, 2004.

[28] J. S. Yeoh, R. van Os, E. Weersing et al., "Fibroblast growth factor-1 and -2 preserve long-term repopulating ability of hematopoietic stem cells in serum-free cultures," Stem Cells, vol. 24, no. 6, pp. 1564-1572, 2006.

[29] M. Seandel, J. M. Butler, H. Kobayashi et al., "Generation of a functional and durable vascular niche by the adenoviral E4ORF1 gene," Proceedings of the National Academy of Sciences of the United States of America, vol. 105, no. 49, pp. 19288 19293, 2008.

[30] J. M. Butler, H. Kobayashi, and S. Rafii, "Instructive role of the vascular niche in promoting tumour growth and tissue repair by angiocrine factors," Nature Reviews Cancer, vol. 10, no. 2, pp. 138-146, 2010.

[31] H. Kobayashi, J. M. Butler, R. O’Donnell et al., "Angiocrine factors from Akt-activated endothelial cells balance selfrenewal and differentiation of haematopoietic stem cells," $\mathrm{Na}$ ture Cell Biology, vol. 12, no. 11, pp. 1046-1056, 2010.

[32] I. B. Mazo, J. C. Gutierrez-Ramos, P. S. Frenette, R. O. Hynes, D. D. Wagner, and U. H. von Andrian, "Hematopoietic progenitor cell rolling in bone marrow microvessels: parallel contributions by endothelial selectins and vascular cell adhesion molecule," Journal of Experimental Medicine, vol. 188, no. 3, pp. 465-474, 1998.

[33] C. Roca and R. H. Adams, "Regulation of vascular morphogenesis by Notch signaling," Genes and Development, vol. 21, no. 20, pp. 2511-2524, 2007.

[34] A. W. Duncan, F. M. Rattis, L. N. DiMascio et al., "Integration of Notch and Wnt signaling in hematopoietic stem cell maintenance," Nature Immunology, vol. 6, no. 3, pp. 314-322, 2005.

[35] M. Wu, H. Y. Kwon, F. Rattis et al., "Imaging hematopoietic precursor division in real time," Cell Stem Cell, vol. 1, no. 5, pp. 541-554, 2007.

[36] B. Varnum-Finney, L. Xu, C. Brashem-Stein et al., "Pluripotent, cytokine-dependent, hematopoietic stem cells are immortalized by constitutive Notch1 signaling," Nature Medicine, vol. 6, no. 11, pp. 1278-1281, 2000.

[37] B. K. Hadland, S. S. Huppert, J. Kanungo et al., "A requirement for Notch1 distinguishes 2 phases of definitive hematopoiesis during development," Blood, vol. 104, no. 10, pp. 3097-3105, 2004.

[38] T. Sugiyama, H. Kohara, M. Noda, and T. Nagasawa, "Maintenance of the hematopoietic stem cell pool by CXCL12-CXCR4 chemokine signaling in bone marrow stromal cell niches," Immunity, vol. 25, no. 6, pp. 977-988, 2006.

[39] K. Parmar, P. Mauch, J. A. Vergilio, R. Sackstein, and J. D. Down, "Distribution of hematopoietic stem cells in the bone marrow according to regional hypoxia," Proceedings of the National Academy of Sciences of the United States of America, vol. 104, no. 13, pp. 5431-5436, 2007.

[40] T. A. Venezia, A. A. Merchant, C. A. Ramos et al., "Molecular signatures of proliferation and quiescence in hematopoietic stem cells," PLoS Biology, vol. 2, no. 10, p. e301, 2004.

[41] M. Rehn, A. Olsson, K. Reckzeh et al., "Hypoxic induction of vascular endothelial growth factor regulates murine hematopoietic stem cell function in the low-oxygenic niche," Blood, vol. 118, no. 6, pp. 1534-1543, 2011.

[42] C. R. Walkley, J. M. Shea, N. A. Sims, L. E. Purton, and S. $\mathrm{H}$. Orkin, "Rb regulatesinteractions between hematopoietic stem cells and their bone marrow microenvironment," Cell, vol. 129, no. 6, pp. 1081-1095, 2007.

[43] J. J. Lataillade, O. Pierre-Louis, H. C. Hasselbalch et al., "Does primary myelofibrosis involve a defective stem cell niche? from concept to evidence," Blood, vol. 112, no. 8, pp. 3026-3035, 2008.

[44] C. M. Edwards, J. R. Edwards, S. T. Lwin et al., "Increasing wnt signaling in the bone marrow microenvironment inhibits the development of myeloma bone disease and reduces tumor burden in bone in vivo," Blood, vol. 111, no. 5, pp. 2833-2842, 2008.

[45] M. H. Raaijmakers, S. Mukherjee, S. Guo et al., "Bone progenitor dysfunction induces myelodysplasia and secondary leukaemia," Nature, vol. 464, no. 7290, pp. 852-857, 2010.

[46] E. Passegué, C. H. M. Jamieson, L. E. Ailles, and I. L. Weissman, "Normal and leukemic hematopoiesis: are leukemias a stem cell disorder or a reacquisition of stem cell characteristics?" Proceedings of the National Academy of Sciences of the United States of America, vol. 100, no. 1, pp. 11842-11849, 2003.

[47] A. Colmone, M. Amorim, A. L. Pontier, S. Wang, E. Jablonski, and D. A. Sipkins, "Leukemic cells create bone marrow niches that disrupt the behavior of normal hematopoietic progenitor cells," Science, vol. 322, no. 5909, pp. 1861-1865, 2008.

[48] M. Ninomiya, A. Abe, A. Katsumi et al., "Homing, proliferation and survival sites of human leukemia cells in vivo in immunodeficient mice," Leukemia, vol. 21, no. 1, pp. 136-142, 2007.

[49] J. W. Hussong, G. M. Rodgers, and P. J. Shami, "Evidence of increased angiogenesis in patients with acute myeloid leukemia," Blood, vol. 95, no. 1, pp. 309-313, 2000.

[50] S. Dias, K. Hattori, Z. Zhu et al., "Autocrine stimulation of VEGFR-2 activates human leukemic cell growth and migration," Journal of Clinical Investigation, vol. 106, no. 4, pp. 511-521, 2000.

[51] T. Padró, R. Bieker, S. Ruiz et al., "Overexpression of vascular endothelial growth factor (VEGF) and its cellular receptor KDR (VEGFR-2) in the bone marrow of patients with acute myeloid leukemia," Leukemia, vol. 16, no. 7, pp. 1302-1310, 2002.

[52] P. Koistinen, T. Siitonen, P. Mäntymaa et al., "Regulation of the acute myeloid leukemia cell line OCI/AML-2 by endothelial nitric oxide synthase under the control of a vascular endothelial growth factor signaling system," Leukemia, vol. 15, no. 9, pp. 1433-1441, 2001.

[53] A. Aicher, C. Heeschen, C. Mildner-Rihm et al., "Essential role of endothelial nitric oxide synthase for mobilization of stem and progenitor cells," Nature Medicine, vol. 9, no. 11, pp. 13701376, 2003.

[54] T. Matsunaga, N. Takemoto, T. Sato et al., "Interaction between leukemic-cell VLA-4 and stromal fibronectin is a decisive factor for minimal residual disease of acute myelogenous leukemia," Nature Medicine, vol. 9, no. 9, pp. 1158-1165, 2003.

[55] R. Möhle, M. Schittenhelm, C. Failenschmid et al., "Functional response of leukaemic blasts to stromal cell-derived factor-1 correlates with preferential expression of the chemokine receptor CXCR4 in acute myelomonocytic and lymphoblastic leukaemia," British Journal of Haematology, vol. 110 , no. 3, pp. 563-572, 2000. 
[56] E. J. Rombouts, B. Pavic, B. Löwenberg, and R. E. Ploemacher, "Relation between CXCR-4 expression, Flt3 mutations, and unfavorable prognosis of adult acute myeloid leukemia," Blood, vol. 104, no. 2, pp. 550-557, 2004.

[57] S. Konoplev, G. Z. Rassidakis, E. Estey et al., "Overexpression of CXCR4 predicts adverse overall and event-free survival in patients with unmutated FLT3 acute myeloid leukemia with normal karyotype," Cancer, vol. 109, no. 6, pp. 1152-1156, 2007.

[58] S. Tavor, I. Petit, S. Porozov et al., "CXCR4 regulates migration and development of human acute myelogenous leukemia stem cells in transplanted NOD/SCID mice," Cancer Research, vol. 64, no. 8, pp. 2817-2824, 2004.

[59] L. Jin, K. J. Hope, Q. Zhai, F. Smadja-Joffe, and J. E. Dick, "Targeting of CD44 eradicates human acute myeloid leukemic stem cells," Nature Medicine, vol. 12, no. 10, pp. 1167-1174, 2006.

[60] M. B. Meads, R. A. Gatenby, and W. S. Dalton, "Environmentmediated drug resistance: a major contributor to minimal residual disease," Nature Reviews Cancer, vol. 9, no. 9, pp. 665674, 2009.

[61] L. A. Milner, R. Kopan, D. I. Martin, and I. D. Bernstein, "A human homologue of the Drosophila developmental gene, Notch, is expressed in CD34+ hematopoietic precursors," Blood, vol. 83, no. 8, pp. 2057-2062, 1994.

[62] K. Ohishi, B. Varnum-Finney, and I. D. Bernstein, "Delta1 enhances marrow and thymus repopulating ability of human CD34+CD38- cord blood cells," Journal of Clinical Investigation, vol. 110, no. 8, pp. 1165-1174, 2002.

[63] C. Delaney, B. Varnum-Finney, K. Aoyama, C. Brashem-Stein, and I. D. Bernstein, "Dose-dependent effects of the Notch ligand Delta1 on ex vivo differentiation and in vivo marrow repopulating ability of cord blood cells," Blood, vol. 106, no. 8 , pp. 2693-2699, 2005.

[64] X. Xu, Y. Zhao, M. Xu et al., "Activation of Notch signal pathway is associated with a poorer prognosis in acute myeloid leukemia," Medical Oncology. In press.

[65] J. E. Karp, I. Gojo, R. Pili et al., "Targeting vascular endothelial growth factor for relapsed and refractory adult acute myelogenous leukemias: therapy with sequential $1-\beta-d-$ arabinofuranosylcytosine, mitoxantrone, and bevacizumab," Clinical Cancer Research, vol. 10, no. 11, pp. 3577-3585, 2004. 

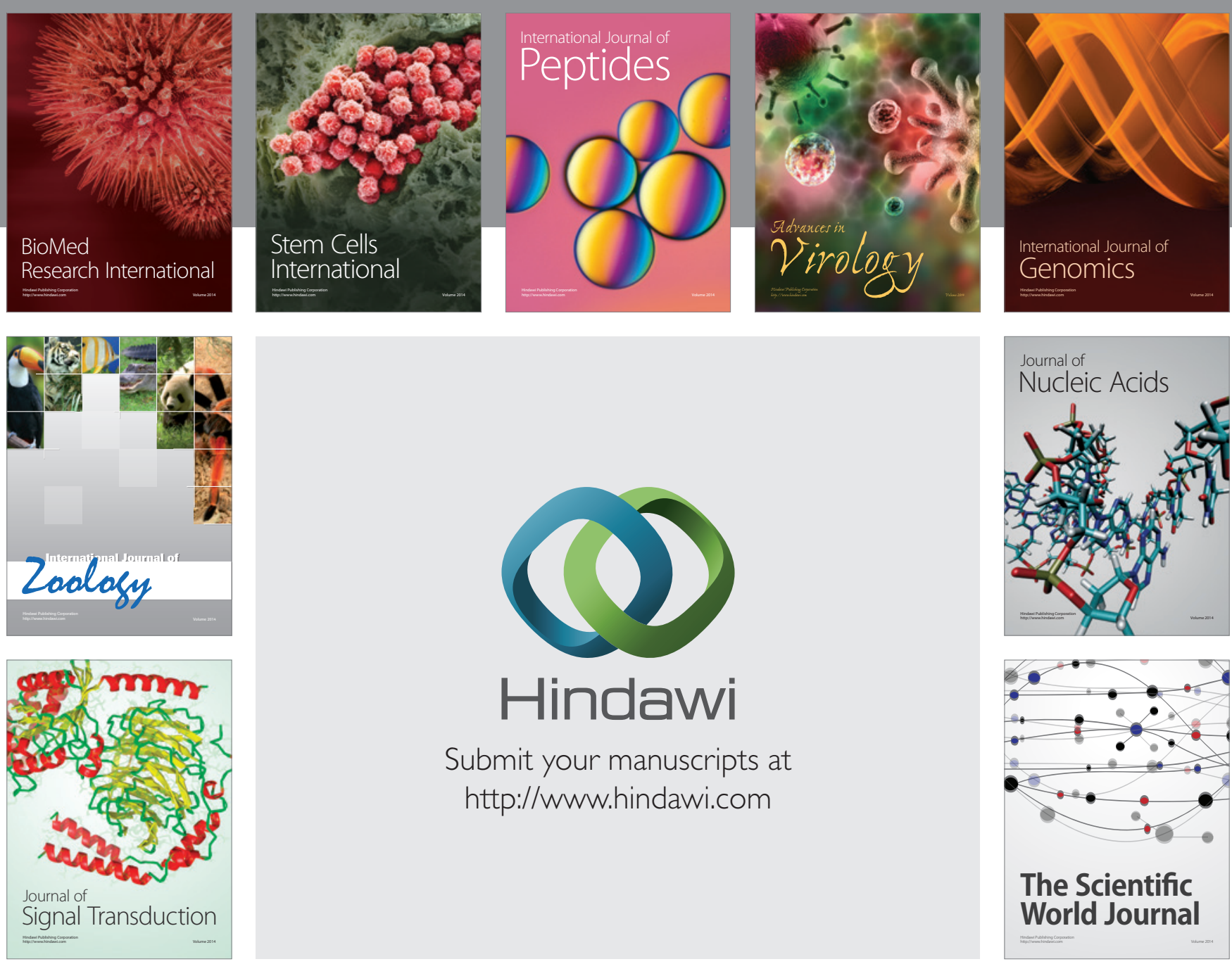

Submit your manuscripts at

http://www.hindawi.com
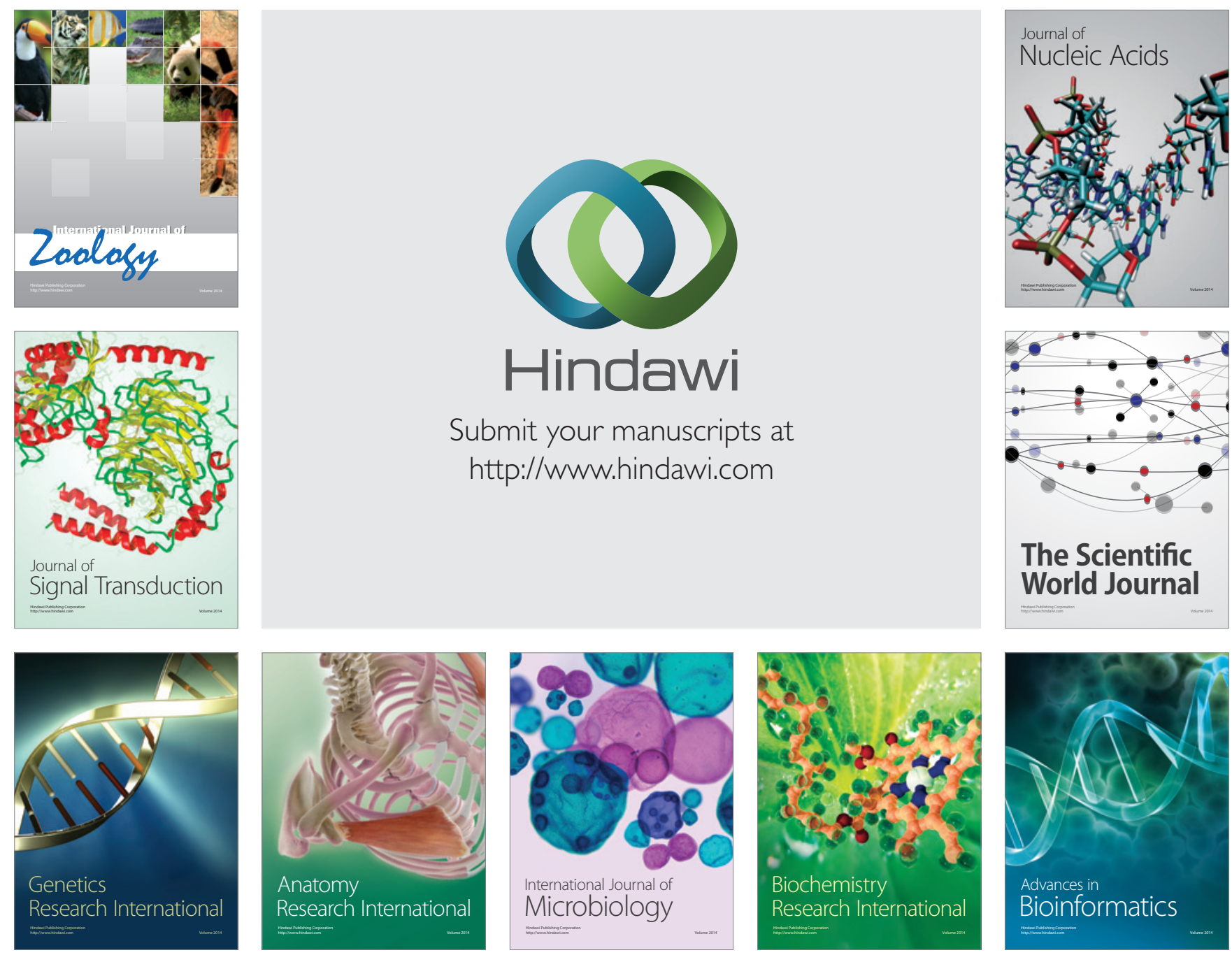

The Scientific World Journal
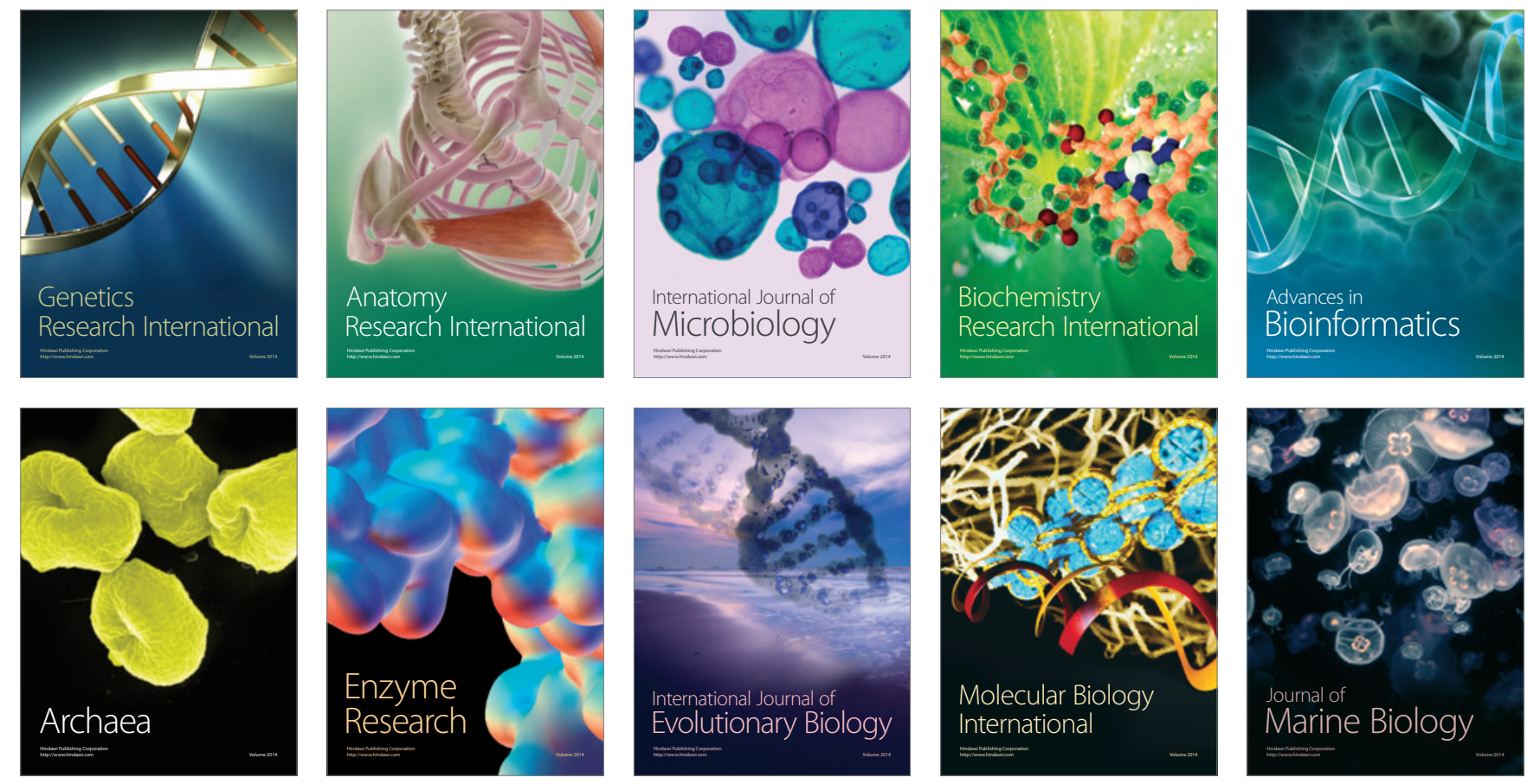